\title{
Hemorrhage from the Pancreatic Duct Caused by a Splenic Vein Fistula
}

We describe here a case of bleeding from the pancreatic duct due to a splenic vein fistula caused by chronic pancreatitis.

A 54-year-old male was admitted to our hospital in August 1987 with hematemesis and melena. Gastrointestinal endoscopy did not reveal a bleeding site, and the hemorrhage subsided with conservative treatment. The patient suffered further bleeding episodes, some with abdominal pain, occurring at monthly intervals from then on. On each of these occasions he was hospitalized in a local hospital. In May 1988, the patient returned to our hospital with signs of gastrointestinal hemorrhage.

Gastrointestinal endoscopy revealed fresh blood oozing from the papilla of Vater. During ERCP examination, the pancreatic duct was found to be enlarged, and it showed irregularities within the

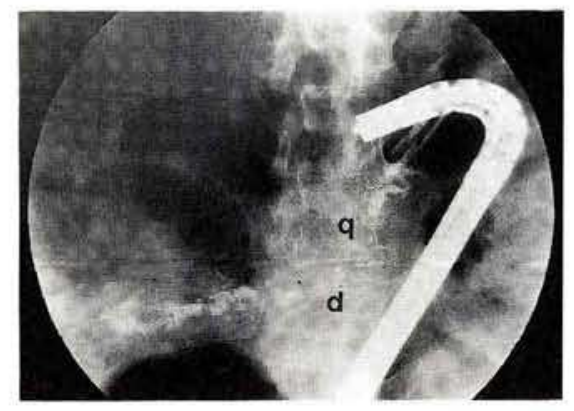

Figure 1: An enlarged pancreatic duct $(p)$ with intralumina blood clots (b). 
duct due to blood clots (Figure 1). The patient underwent surgery, with subtotal left resection of the pancreas and splenectomy being performed. He recovered well, and has been free of symptoms since. Histological examination of the surgical material showed a fistula between the splenic vein and the pancreatic duct. The pancreatic duct was dilated $(8 \mathrm{~mm})$ and filled with thrombotic masses (Figure 2). Reviewing the literature (1-4), and in our experience, pancreatic duct bleeding should be suspected if there is recurrent gastrointestinal hemorrhage without any obvious cause combined with a history of chronic pancreatitis and colicky epigastric pain during bleeding episodes. These clinical findings are explained by rapid filling of the pancreatic duct with blood, leading to a dilatation causing pain and probably an increase of serum amylase. The increasing pressure in the duct and formation of blood clots seals the fistula, and the bleeding subsides until the lysis of clots after a variable period of time leads to a recurrence of the process. Thus, bleeding episodes happen repeatedly for months or even years ( 3 , 4). In cases of pancreatic duct bleeding, most researchers agree that the method of choice for definitive treatment is surgery.

\section{References}

1 van Rooyen $W$, van Blankenstein $M$, Schattenkerk ME et al.: Hemorrhage from the pancreatic duct: a rare form of upper gastrointestinal bleeding. Br. J. Surg. 1984; 71: 137-140.

2 Bivins BA, Sachatello CR, Chuang VP et al.: Hemosuccus pancreaticus. Arch. Surg. 1978; 113: 751-753.

3 Cahow CE, Gusberg RJ, Gottlieb LJ: Gastrointestinal hemorrhage from pseudoaneurysms in pancreatic pseudocysts. Am. J. Surg. 1982; 145: 534-541.

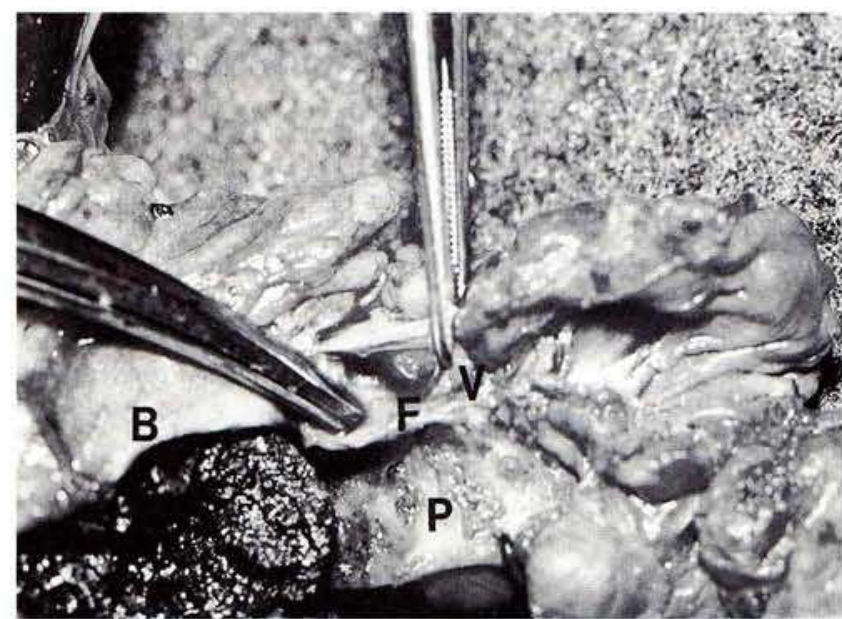

Figure 2: Blood clots (B) in the pancreatic duct. F: fistula, $\mathrm{P}$ : pancreatic duct, V: splenic vein.

4 Stanley JC, Frey CF, Miller TA et al: Major arterial hemorrhage. Arch. Surg. 1976; 111: 435-440.

K. Glaser ${ }^{\text {, }}$ E. Bodner ${ }^{1}$, G. Wetscher ${ }^{\text {, }}$, A. Weger ${ }^{2}$, J. Tschmelitsch ${ }^{\prime}, P$. Klingler ${ }^{\prime}, R$. Pointner ${ }^{\prime}$

${ }^{1}$ Second Department of Surgery, ${ }^{2}$ Department of Pathology, University of Innsbruck, Anichstrasse 35, 6020 Innsbruck, Austria 